\title{
Improving Earth-like planets' detection with an ELT: the differential radial velocity experiment
}

\author{
P. Riaud ${ }^{1,2}$ and J. Schneider ${ }^{3}$ \\ 1 LESIA, Observatoire de Paris-Meudon, 5 place J. Janssen, 92195 Meudon, France \\ e-mail: riaud.pierre@obspm.fr \\ 2 Université de Liège, 17 Allée du 6 Août, 4000 Sart Tilman, Belgium \\ e-mail: riaud@astro.ulg.ac.be \\ ${ }^{3}$ LUTH, Observatoire de Paris-Meudon, 5 place J. Janssen, 92195 Meudon, France \\ e-mail: jean.schneider@obspm.fr
}

Received 11 January 2007 / Accepted 17 February 2007

\begin{abstract}
Context. Direct detection of Earth-like planets around a nearby star is extremely difficult for ground-based telescopes equipped with the eXtreme Adaptative Optics system.

Aims. We propose to combine high-precision radial velocity with an ELT to detect extrasolar planets with a feasible coronagraph. Methods. In this context, we investigated the detectability of Earth-like extrasolar planets with an ELT of $42 \mathrm{~m}$ equipped with the spectro-coronagraphic instrument in an eXtrem Adaptive Optics system. Those stellar residuals left uncorrected by the XAO wavefront correction (Strehl ratio $>85 \%$ ) system need to be calibrated. We propose calibrating them by the radial velocity measurement of the planet in a multiple spectrum correlation process.

Results. This method allows detection of Terrestrial planets with an ELT but requires a long exposure time $(\approx 200 \mathrm{~h})$

Conclusions. The technique overcomes the main coronagraphic limitation due to the pinned and the low variable speckles and opens a way to detect directly exoplanets with large ground-based telescopes equipped with an efficient XAO system.
\end{abstract}

Key words. stars: planetary systems - techniques: spectroscopic - techniques: radial velocities - astrobiology

\section{Introduction}

The detection and characterization of Earth-like extrasolar planets is one of the major goals of astronomy for the coming decades. It is in particular one of a few main objectives of ESA (ESA BR-247) and of the ESO ELT project (Brunetto et al. 2004). It is presently not clear which is the better approach, ground or space (see Direct Imaging of Exoplanets: Riaud et al. 2002). The difficulties of detecting Earth-like extrasolar planets with an ELT with various coronagraphic schemes have been investigated, for example, by Le Louarn et al. (2004), Cavarroc et al. (2006). These studies show that the detection will be extremely difficult. The major limitation of the Earth-like planet detection by an ELT is the speckle level given by adaptive optics residuals through the coronagraphic device. In the visible band best case, we obtain a residual contrast ratio of about 1000 to 10000 compared to $10^{10}$ without coronagraph (Cavarroc et al. 2006).

In this paper we propose an improved approach based on the difference in radial velocity between the planet and the parent star. The idea is to extract the radial velocity of the planet from the residual stellar flux halo. The paper is organized as follows. Section 2 presents the principle of our method, and Sect. 3 shows different spectral band optimisation for various stellar types. In Sect. 4, we calculate the gain obtained with the "(Differential Radial Velocity Experiment" (DRiVE) on a realistic coronagraphic device (Riaud et al. 2001; Mawet et al. 2005). A signal-to-noise ratio analysis is also given at the end of this section with numerical simulations of multicorrelation analysis
Finally, we discuss a possible technical implementation in the visible with an Integral Field Unit Spectrograph.

\section{Principle of the method}

The principle of the DRiVE instument is to improve the Earthlike planet signature in the high background of the stellar residual flux thanks to the planet radial velocity information. Consider an image of a planetary system with one planet on a circular orbit, for simplicity. The planet image will be imbedded in a collection of speckles that are generally 100 to 10000 times brighter. But, unless the planet orbit lies in the sky plane, it will have a different radial velocity from the star. For a planet at a distance $a$ from the central star, the velocity difference is $V_{\mathrm{r}}=$ $\sqrt{G \cdot M_{\mathrm{s}} / a} \cdot \sin i$ at maximum elongation: $M_{\mathrm{S}}$ is the star mass, $i$ the inlination of the planet orbit and $G$ the gravitationnal constant. A planet at $1 \mathrm{AU}$ around a $1 M_{\odot}$ star presents a maximum radial velocity of $V_{\mathrm{r}}=30 \sin i \mathrm{~km} \mathrm{~s}^{-1}$. The spectrum of the planet will therefore be shifted by a quantity $\Delta \lambda / \lambda=\Delta V_{\mathrm{r}} / c=10^{-4}$ for $\sin i=1$. If we can manage a spectro-imager with a spectral resolution of $>2 \times 10^{4}$, i.e. with a minimum spectral channels of $0.5 \AA$ at $1 \mu \mathrm{m}$, the planet signal should be fairly distinguishable from the stellar speckles.

\section{Spectral band optimisation}

For different stellar types, the habitable zone (HZ) depends on the stellar photosphere temperature (Riaud et al. 2002), showing variations in the distance according to the stellar type. The radial 


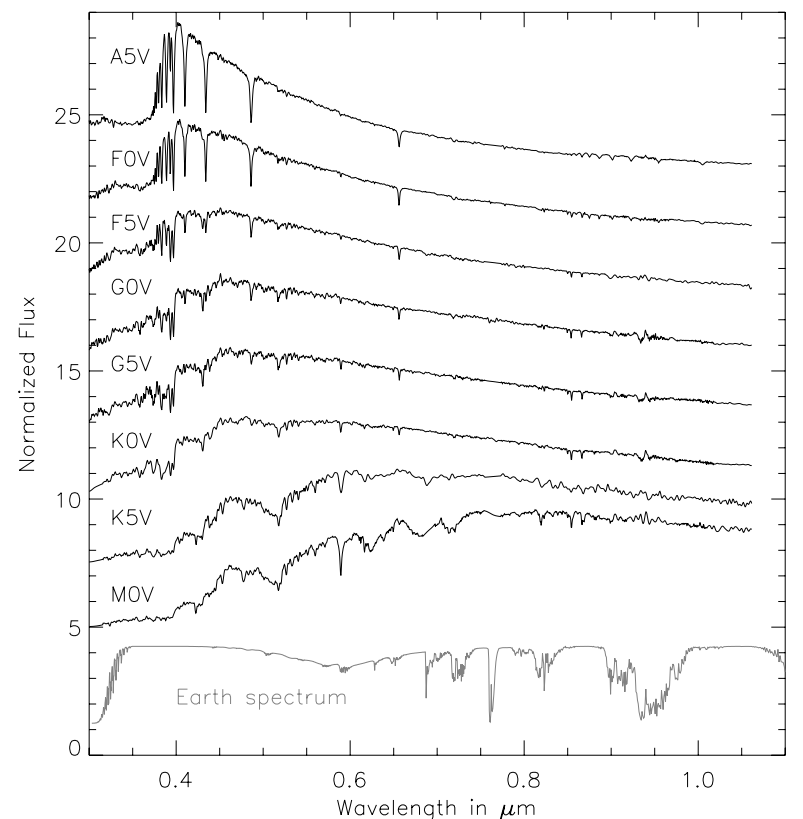

Fig. 1. The figure shows various stellar spectra for the main sequence stars (A5 to M0). Below, the grey curves show the Earth spectrum with various absorption bands like $\mathrm{H}_{2} \mathrm{O}, \mathrm{O}_{2}$ and the Chappuis band of $\mathrm{O}_{3}$ around $0.6 \mu \mathrm{m}$ (Arnold et al. 2002).

velocity of the planet in the life zone thus depends on the stellar parameters (temperature and mass). Under these conditions, we must optimize the spectral band according to the stellar parameters.

Table 1 summarizes the maximum radial velocity of the planet and the working spectral band. Two cases show up, (i) the hot stars $\left(T_{\mathrm{s}}>25000 \mathrm{~K}\right)$ where all lines in absorption are broadened thermally and (ii) cold stars like M 5 where the spectrum presents large $\mathrm{TiO}$ bands. In the first case, where the thermal behavior is important, the full width half-maximum of spectral lines is given by the classical formula: $\sqrt{2 k T / m_{\mathrm{H}}}$ with $k$ the Boltzmann constant and $m_{\mathrm{H}}$ the mass of hydrogen atom. All stars in this study present a thermal velocity of about $7 \mathrm{~km} \mathrm{~s}^{-1}$ for a M 5 star to $12 \mathrm{~km} \mathrm{~s}^{-1}$ for a A 5 star. These values are always lower than the radial velocity by a factor 2 to 5 . The second problem concerns the strong, wide absorbing bands in the red part of the visible spectrum $(\lambda>700 \mathrm{~nm})$ like the TiO bands. This effect is very important for the M 5 stellar type, and the methods seem to be very limited. (The simultations in Sect. 4 will confirm this assumption.) The spectral bandwidth parameter in the Table 1 shows the spectral band optimization needed for all spectral types. The optimal spectral bandwidth is $650-850 \mathrm{~nm}$ for all type stars, except M 5 and also for those corresponding to the best quantum efficiency of a red enhanced CCD ship. Finally, some hot stars present strong equatorial rotation. It leads to a line broadening of 5 to $100 \mathrm{~km} \mathrm{~s}^{-1}$ for a few peculiar stars. Table 1 gives some parameters for a wide range of spectral types.

\section{Background and signal-to-noise ratio at high spectral resolution}

In this section, we are interested in the signal-to-noise ratio at high spectral resolution in the presence of different noises. For a telescope of diameter $D$, a star of magnitude $m$, a planet-to-star flux ratio $C_{\mathrm{r}}$ and a detection efficiency $\epsilon$, the number of photoelectrons detected from the planet in the $V$ band is

$N_{\text {ph-el }}=\epsilon 10^{3} 10^{-0.4 m} C_{\mathrm{r}}(D / 1 \mathrm{~cm})^{2} / \AA / \mathrm{s}$.

For a typical solar-type star with $m=4.85$, an overall efficiency of $15 \%$ and a planet-to-star ratio $C_{\mathrm{r}}=1.5 \times 10^{-10}$, one would thus detect 16 photons/hour in a spectral resolution element with $\delta \lambda=1 \AA$ with $D=42 \mathrm{~m}$ ELT.

When searching for a planet by direct coronagraphic imaging, one does not know where the planet lies in the image (unless some information is provided by radial velocity or astrometric measurements of the parent star). On a given point of the image there are, in addition to the planet itself, two contributions to the flux: stellar residuals after the coronagraphic device and exo-zodiacal light.

\subsection{Stellar residual contribution under $X A O$ implementation}

To improve the exo-Earth planet detection, it is nessessary to use the complex eXtrem Adaptive Optics system with a large number of actuators. Table 2 shows all the adaptive optics characteristics used in the numerical simulations. The DRiVE spectro-coronagraphic instrument must be implemented with this powerful XAO system. In these conditions, we performed a coronagraphic simulation with an ELT $(D=42 \mathrm{~m})$ the presence of low phase residuals after correction of the atmospheric turbulence with high-order adaptive optics (XAO). Indeed, in the $V$ band, and to some extent, in the $R$ band, high-order adaptive optics with $10^{6}$ actuators could provide a high Strehl ratio of about $85-90 \%$ in the $R$ band. We use a simplified model of AO: the corrected phase screen is given by a simple PSD (power spectral density) on a one-layer model. A simulation with three layers of turbulence and scintillation behavior consumes CPU and it shows no difference with this simplified model. This effect can be easily explained by the fact that the final coronagraphic image is an average of 1024 speckle realizations. The numerical simulations are performed with large arrays $(4096 \times 4096)$ to minimize the aliazing effect and allow sufficient focal plane sampling for a coronagraphic study. All AO parameters are listed in Table 2.

The high Strehl ratio obtained in this configuration is not sufficient for a direct exo-earth detection. We also included a well-chosen coronagraphic device in our simulations: a mixed configuration of a large Lyot coronograph (Lyot 1939) and a phase mask like the AGPM coronagraph (Mawet et al. 2005). The $12 \lambda / D$ diameter Lyot mask provides an important attenuation factor on the stellar peak. The AGPM phase mask increases the rejection factor and allows a halo smoothing in the large angular separation. In the coronagraphic simulations, we consider the gap between each hexagon in the primary mirror and also in the secondary mirror. The non-common path error after the Lyot stop is $0.5 \mathrm{~nm} \mathrm{rms}$ in the numerical simulation. This value seems to be challenging, but a complete optical bench in high homogenous SUPRASIL could fulfill these requirements. The final coronagraphic image presents some narrow peaks due to the ELT mirror structure (hexagonal). For the sake of simplicity, we present two averaged profiles (the minimum and the maximum flux in the coronagraphic image) to allow a total characterization of residual flux after the coronagraphic device. We also show a possible gain after a two-wavelength subtraction process (Marois et al. 2004). In all cases, the stellar residual is always greater than $10^{-9}$, which explains the difficulty detecting Terrestrial planets even with large angular planet separation (Cavarroc et al. 2006). 
Table 1. Stellar parameters for detecting Earth-like planets.

\begin{tabular}{ccccccc}
\hline \hline $\begin{array}{c}\text { Stellar } \\
\text { type }\end{array}$ & $\begin{array}{c}\text { Temperature } \\
\text { in K }\end{array}$ & $\begin{array}{c}\text { Stellar mass } \\
\text { in } M_{\odot}\end{array}$ & $\begin{array}{c}\text { HZ } \\
\text { in AU }\end{array}$ & $\begin{array}{c}\text { radial velocity } \\
\text { in km s }\end{array}$ & $\begin{array}{c}\text { Thermal } \\
\text { broadening in km s }\end{array}$ & $\begin{array}{c}\text { Spectral } \\
\text { bandwith }\end{array}$ \\
\hline A5 & 8650 & 1.85 & 4.27 & 20 & 12 & $\lambda>0.5 \mu \mathrm{m}$ \\
F0 & 7250 & 1.5 & 2.79 & 22 & 10.9 & $\lambda>0.5 \mu \mathrm{m}$ \\
F5 & 6500 & 1.2 & 1.89 & 24 & 10.3 & $\lambda>0.5 \mu \mathrm{m}$ \\
G0 & 5900 & 1.05 & 1.20 & 28 & 9.9 & $\lambda>0.3 \mu \mathrm{m}$ \\
G2 & 5780 & 1. & 1 & 30. & 9.7 & $\lambda>0.3 \mu \mathrm{m}$ \\
G5 & 5600 & 0.95 & 0.89 & 31 & 9.6 & $\lambda>0.45 \mu \mathrm{m}$ \\
K0 & 5250 & 0.85 & 0.64 & 34 & 9.3 & $\lambda>0.55 \mu \mathrm{m}$ \\
K5 & 4400 & 0.65 & 0.41 & 38 & 8.5 & $\lambda>0.7 \mu \mathrm{m}$ \\
M0 & 3900 & 0.55 & 0.24 & 45 & 8 & $\lambda>0.75 \mu \mathrm{m}$ \\
M5 & 3100 & 0.15 & 0.13 & 33 & 7 & Problem with TiO bands \\
\hline
\end{tabular}

Table 2. Phase screen model parameters.

\begin{tabular}{ccc}
\hline \hline Parameters & Paranal & Dome C \\
Seeing at $0.55 \mu \mathrm{m}$ & $0.8^{\prime \prime}$ & $0.24^{\prime \prime}$ \\
$D / r_{0}$ & 280 & 84 \\
Outerscale of turbulence $L_{0}$ & $25 \mathrm{~m}$ & $>100 \mathrm{~m}$ \\
Number of actuators & $\approx 10^{6}$ & $\approx 10^{5}$ \\
Pupil sampling & $7.5 \mathrm{~cm} / \mathrm{pixel}$ & $7.5 \mathrm{~cm} / \mathrm{pixel}$ \\
Correction frequency & $5 \mathrm{Khz}$ & $1 \mathrm{KHz}$ \\
Tip-tilt correction & $0.5 \mathrm{mas} \mathrm{rms}$ & $0.5 \mathrm{mas} \mathrm{rms}$ \\
Wavelength & $0.7-0.8 \mu \mathrm{m}$ & $0.7-0.8 \mu \mathrm{m}$ \\
Average Strehl ratio @ 750 nm & $\approx 85-90 \%$ & $\approx 85-90 \%$ \\
Number of speckle realizations & $1024 \approx 0.2 \mathrm{~s}$ & $1024 \approx 1 \mathrm{~s}$ \\
\hline
\end{tabular}

We denote $f_{\mathrm{R}}(\rho)$ as the ratio of stellar residual flux over the planet flux obtained after the coronagraphic device. If we take an averaged contrast of $C_{\mathrm{r}}=1,5 \times 10^{-10}$ for an Earth-like planet, then $f_{\mathrm{R}}(\rho)=1300$ to 3300 (see Fig. 2). Then, the signal-to-noise ratio in a spectral channel with a width $\Delta \lambda$ is

$S / N \approx \sqrt{N_{\mathrm{ph}-\mathrm{el}}(\Delta \lambda) \cdot t / f_{\mathrm{R}}(\rho)}$.

A signal-to-noise ratio $(S / N)$ of 5 per channel is reached for an exposure time of $990 \mathrm{~h}$ for $f_{\mathrm{R}}(\rho)=1300$ and $2510 \mathrm{~h}$ for $f_{\mathrm{R}}(\rho)=$ 3300.

For $N_{\mathrm{L}}$ spectral lines, the final $S / N$ is

$S / N \approx \sqrt{N_{\mathrm{ph}-\mathrm{el}}(\Delta \lambda) \cdot t / f_{\mathrm{R}}(\rho)} \sqrt{N_{\mathrm{L}}}$

reducing the exposure time to $31.3 \mathrm{~h}$ and $79.5 \mathrm{~h}$ for $N_{\mathrm{L}}=1000$ and $f_{\mathrm{R}}(\rho)=1300$ to 3300 , respectively.

\subsection{Exo-zodiacal contribution}

Before discussing this contribution, let us clarify the geometrical notations. The exo-zodiacal dust is assumed, for simplicity, to be distributed uniformly in a disk with a radius $R_{\mathrm{D}}$, thickness $d_{Z}$, and inclination $i$. Let $\boldsymbol{n}$ (unit vector) be the projection on the sky of the perpendicular to exo-zodiacal plane.

Let $(\boldsymbol{x}, \boldsymbol{y})$ be an orthogonal base vector centered on the parent star in the focal plane. Let consider a resolution element in the image with an angular size $\Delta \alpha=\lambda / D$ around a current line of sight (containing the planet or not). Here $\theta$ is the angle position of a resolution element in the image plane $(\theta=\operatorname{Atan}(\Delta y / \Delta x))$ where $\Delta x$ and $\Delta y$ are the cartesian position. Figure 3 shows the exo-zodiacal disk configuration and the projection in the focal plane.

Let $f_{\mathrm{ZL}}$ be the total flux ratio $F_{\mathrm{Zodi}} / F_{\mathrm{pl}}$ between the exozodiacal light integrated on a resolution element and the planet

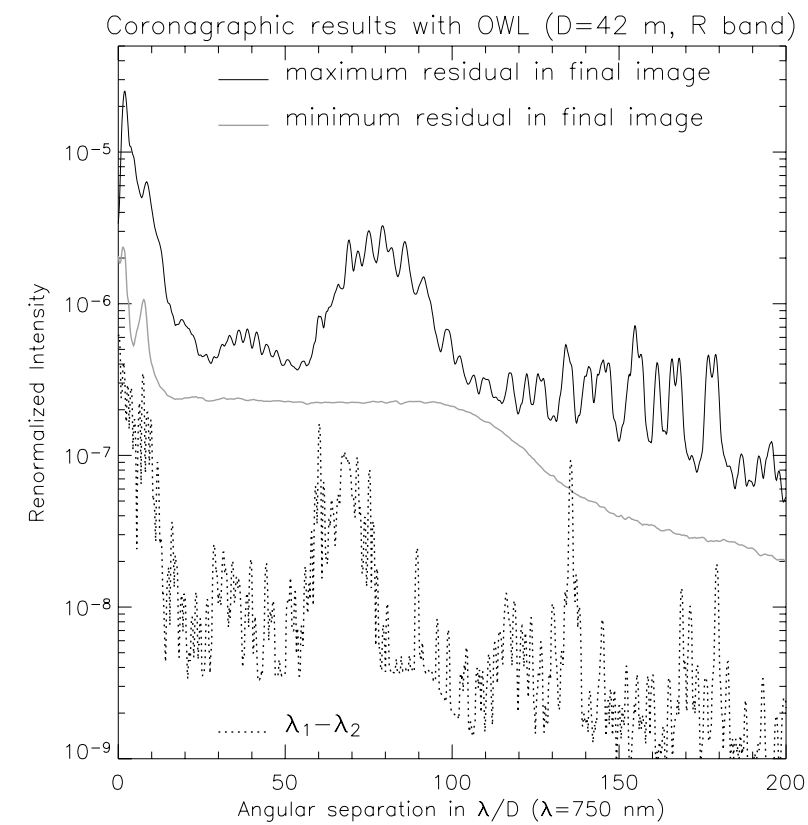

Fig. 2. Radial coronographic profile obtained with the hybrid configuration Lyot+AGPM in $R$ band (spectral bandwidth $650-850 \mathrm{~nm}$ ) with a very good Strehl ratio $(\approx 90 \%)$. The diaphragm in the relayed pupil plane was chosen to be 0.8 times the geometric pupil diameter. This result directly allow the stellar residuals in the final coronagraphic image. The solid line shows the maximum residual in the final coronagraphic image. The grey line shows the minimum starlight residuals. The dotted line shows the averaged profile after two wavelengths subtraction. The radial smearing of the speckles is naturally included by the multiwavelength analysis.

flux. The $f_{\mathrm{ZL}}$ background has a spectrum that depends on the observing direction relative to the parent star. We assume the pessimistic case where the exo-zodi disk and the planet orbit are coplanar. In the pencil beam with a width $\Delta \alpha$ around the observing direction, each line of sight corresponds to a different radial velocity, hence to a different Doppler shift. The mathematical development is given for a Dirac-like spectal feature. The global effect of the exo-zodiacal light will be computed by the entire stellar spectrum convolution .

To discuss the zodi-to-planet flux ratio received in the current image resolution element $\Delta \alpha$, we consider first case the general:

\subsubsection{Disk with an inclination $i$}

In the disk plane (see Fig. 3), we choose the following geometrical notations: $x x$ is the integration variable on the line of sight, 


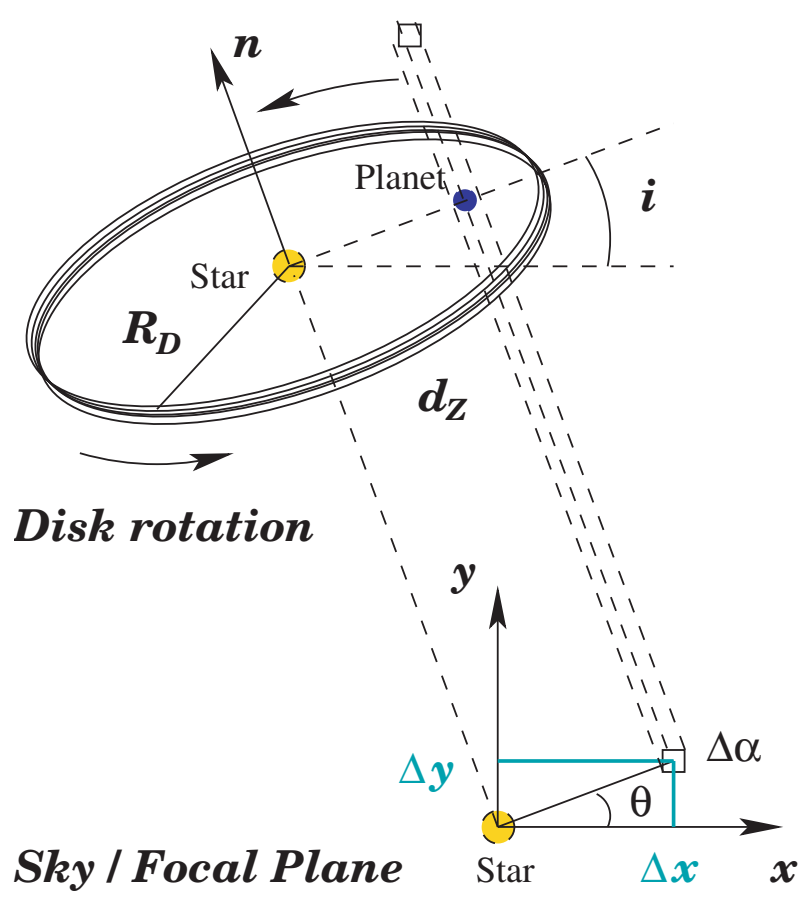

Fig. 3. Scheme for the geometry of exo-zodiacal dust adopted for the calculation of the radial velocity in Sect. 4.2. $d_{Z}$ is the exo-zodiacal disk, $i$ inclination, and $R_{\mathrm{D}}$ the maximum extension of the disk around the observed star $\left(R_{\mathrm{D}}=6 \mathrm{AU}\right) . \Delta \alpha$ is the surface of the image resel (resolution element) on the CCD detector.

$y y$ is the distance of this line to the cental star, $d_{*}$ the star distance in parsec. The velocity $V(x x)$ of the dust between the two limits of the disk $-x_{\mathrm{D}}$ and $x_{\mathrm{D}}$ is given by:

$V(x x)=\sqrt{\frac{G M_{*}}{a(x x)}} \cdot \cos (i)$
$\cos (i)=y y / \sqrt{x x^{2}+y y^{2}}$
$V(x x) / c=\sqrt{G M_{*}} \cdot \frac{y y}{c \cdot\left(x x^{2}+y y^{2}\right)^{3 / 4}}$

$\left(\frac{\Delta \lambda}{\lambda}\right)_{\mathrm{ZL}}=\int_{y_{\mathrm{p}}-\alpha \lambda /(2 . D)}^{y_{\mathrm{p}}+\alpha \lambda /(2 \cdot D)} \int_{-x_{\mathrm{D}}}^{x_{\mathrm{D}}} \frac{\sqrt{G M_{*}} \cdot y y}{c \cdot\left(x x^{2}+y y^{2}\right)^{3 / 4}} \cdot \mathrm{d} x x \cdot \mathrm{d} y y$

$x_{\mathrm{D}}=\sqrt{R_{\mathrm{D}}^{2}-y y^{2}}$

$\alpha=206265 \times d_{*}$.

\subsubsection{Disk seen edge-on}

Different cases exist depending on the intersection of the line of sight of light and the exo-zodiacal disk. We take the most pessimistic case where the disk is seen edge-on. The integration on the $x x$ parameter is easy. Then an individual stellar spectral feature reflected by the zodiacal dust is broadened with a width $\Delta \lambda$ given by

$$
\begin{aligned}
& \left(\frac{\Delta \lambda}{\lambda}\right)_{x x}=\frac{2 \sqrt{G M_{*}} \cdot \sqrt{R_{\mathrm{D}}^{2}-y y^{2}}}{c \cdot \sqrt{y y}} \cdot{ }_{2} F_{1}\left(R_{\mathrm{D}}, y y\right) \\
& { }_{2} F_{1}\left(R_{\mathrm{D}}, y y\right)={ }_{2} F_{1}\left[1 / 2,3 / 4,3 / 2,1-\left(R_{\mathrm{D}}^{2} / y y^{2}\right)\right]
\end{aligned}
$$

where ${ }_{2} F_{1}$ is the Gauss's hypergeometric function. Now we must know the contribution of exo-zodiacal broadering in the resolution element $\Delta \alpha$ (see Fig. 3). This behavior corresponds to the

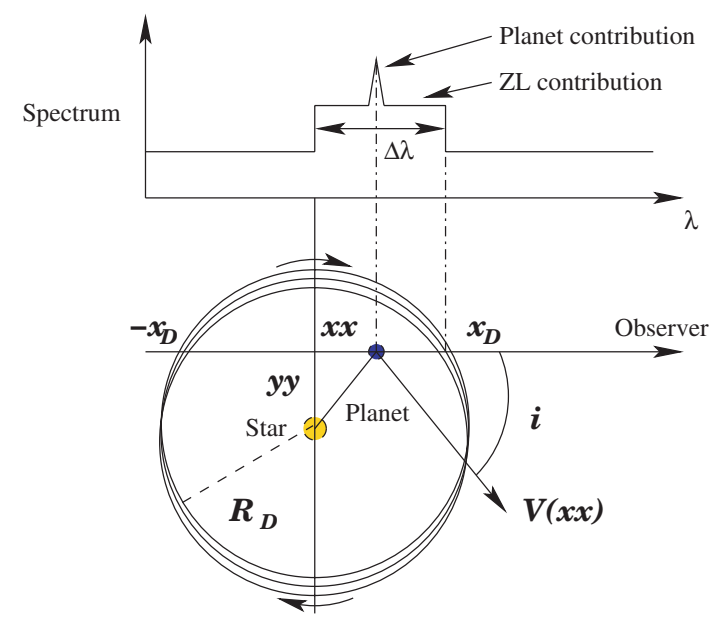

Fig. 4. Bottom figure: geometrical parameters adopted to calculating the exo-zodiacal broadening. $V(x x)$ is the radial velocity, $R_{\mathrm{D}}$ is the extension of the exo-zodiacal cloud around the observed star (we take an external radius of $6 \mathrm{AU}$ ). $x x$ and $y y$ are the two variables where we integrate all the radial velocity of the exo-zodiacal dust. Upper figure: resulting spectrum after exo-zodiacal broadening. The planet features remain present on a large, smooth star spectra.

integration of the $y y$ parameter on the disk thickness centered on planet position $y_{\mathrm{p}}$. This calculation can only be done numerically. Owing to the high angular resolution provided by the ELT, the distance around the planet position $y_{\mathrm{p}}$ is $2 \times 10^{-3} \mathrm{AU}$ and $0.072 \mathrm{AU}$ for a star at 1.34 and $50 \mathrm{pc}$, respectively. In these conditions, the relative weight of contributions to the spectrum for a given spectral line is given by the $Z L_{\Delta \alpha}$ in the beam of angular radius $\Delta \alpha$, where the $Z L_{\Delta \alpha}$ coefficient is the total broadening on a spectral feature in $\Delta \lambda / \lambda$ unit. For a solar system like $Z L$, one has (assuming $R_{\mathrm{D}}=6 A U$ ), $Z L_{\Delta \alpha}=0.015$ for an M5V star at $1.34 \mathrm{pc}$ to 1.6 for an FOV at $50 \mathrm{pc}$. We convolve each stellar spectrum $S_{p}(\lambda)$ given by UVES (Bagnulo et al. 2003) with a kernel proportional to the wavelength. The proportionality factor is given by $Z L_{\Delta \alpha}$, which depends on the distance of the observed star. However, all distances give a large smoothed stellar spectrum without spectral features. In these conditions the exozodiacal broadening effect does not seems to be dominant in the error budget of the DRiVE instument.

We also notice a possible starlight broadening by the stellar rotation. Some relatively hot stars or/and young stars present a high velocity of around $100 \mathrm{~km} \mathrm{~s}^{-1}$ ( $\beta$ Pictoris like stars) but for the main sequence star (ages $>1$ Gyr) with moderate rotation, the width of spectral lines is typically 3 to $8 \mathrm{~km} \mathrm{~s}^{-1}$, significantly lower than the planet Doppler shift (see Table 4).

The planet lying on the same line of sight can have any radial velocity (relative to the star):

$$
\sqrt{G M_{*} / y y} \geq V_{\mathrm{r}} \geq \frac{\sqrt{G M_{*} / R_{\mathrm{D}}} \times y y}{\sqrt{R_{\mathrm{D}} 2+y y^{2}}} .
$$

\subsubsection{Spectra correlation}

Contrary to Bruston (1992), the autocorrelation function $C_{k, p, q}$ is not the simple correlation of a spectrum and the same spectrum with a small shift due to the planetary radial velocity but a multiple spectrum correlation on the large number of pixels. The main idea of DRiVE is to enhance the planetary detection by the cross-correlation spectra. Then, after a proper renormalization between the two spectra on pixel $p$, and on 

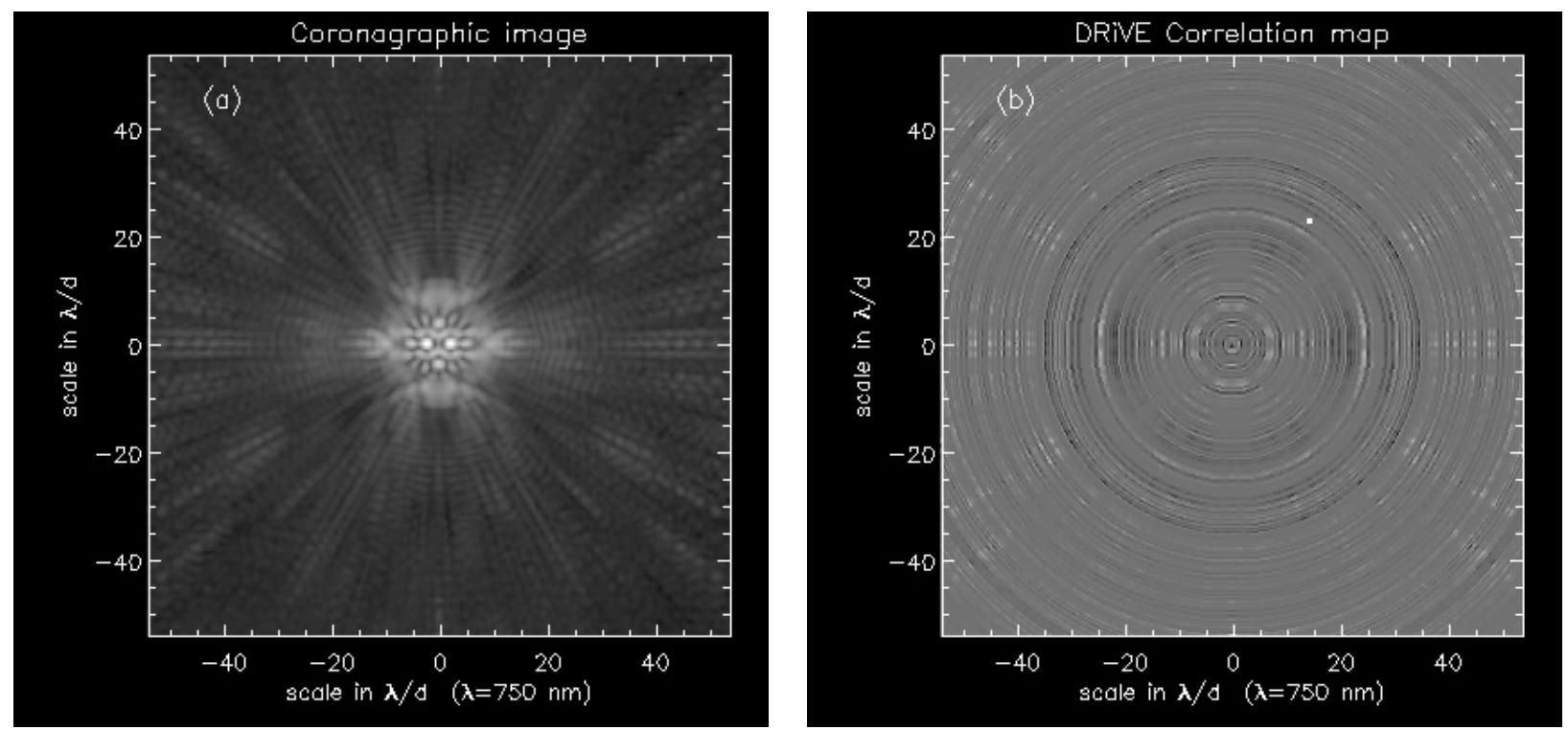

Fig. 5. Left: a) coronagraphic residuals in the focal plane before the high resolution spectrograph in wide band $(650-850 \mathrm{~nm})$. Right: b) the correlation map obtained for the G2V star at $10 \mathrm{pc}$ with an Earth-like planet at $1 \mathrm{AU}$. The total exposure time is $200 \mathrm{~h}$ and the signal-to-noise ratio on the planet peak is 10 . Figure 6 shows the radial correlation.

pixel $q$, the multiple correlation function $C_{k, p, q}$ can be defined by

$C_{k, p, q}=1-\sum_{\lambda}\left(S(\lambda)_{p}-S(\lambda-k)_{q}\right) \cdot S(\lambda-k)_{q}$

where $k$ is the spectral shift due to the expected planetary radial velocity. The multiple correlation function $C_{k, p, q}$ is directly proportional to the difference spectrum between two speckles due to the presence of the planet peak.

To be effective, our algorithm requires a subtraction of spectrum in pixel $p,\left(S(\lambda)_{p}\right)$ by the spectrum in pixel $q,\left(S(\lambda)_{q}\right)$ and also a sufficient structured spectrum with several absorption or emission lines. The previous analysis in Sect. 3 showed the optimal spectral bandwidth $(650-850 \mathrm{~nm})$. In these conditions, the corresponding standard deviation $\sigma_{(k, p, q)}$ becomes

$\sigma_{(k, p, q)}^{2}=\sum_{\lambda} \sum_{p, q} \frac{S(\lambda)_{p}\left(C_{0, p, q} \cdot S(\lambda-k)_{p}-C_{k, p, q} \cdot S(\lambda)_{p}\right)^{2}}{\left(C_{0, p, q} 2-C_{k, p, q} \cdot C_{-k, p, q}\right) 2}$.

With this assumption, a large variance of the $C_{k, p, q}$ function corresponds to a probable planet detection. One limitation could appear in this algorithm is a proper calibration of spectra. The HARPS instrument (Pepe et al. 2003; Lovis et al. 2006) shows very high precision in terms of spectral calibration issues that are quite higher than what is required here $\left(\approx 1 \mathrm{~km} \mathrm{~s}^{-1}\right)$. Finally, to simplify our algorithm, we do not take the speckle smearing effect into account in the spectra correlation. Indeed, all stellar speckles contibution are radially smeared in the final coronagraphic image but not the planet peak (see Fig. 5a). This effect is more complicated in the AO system, because an important contribution is given by a nearly white residual halo. The speckle smearing can be implemented easily in the multiple correlation function $C_{k, p, q}$ by the spectral bandpass limitation between two correlated spectra.

\subsubsection{Signal-to-noise ratio simulations}

In these conditions, to scan possible Earth-like planets around nearby stars $(d<20 \mathrm{pc})$, it is nessary to have a FOV of $1^{\prime \prime}$ in diameter. The last section developed some conditions for fulfill in instrumental constraints. We consider here an observation with an IFU (Prieto \& Vivès 2006; Zamkotsian et al. 2006) on an ELT telescope like the ESO/OWL project (Vérinaud et al. 2006) in high spectroscopic-resolution mode with real stellar spectra templates obtained with the UVES spectrograph $(R \approx 120000)$ on the VLT (Bagnulo et al. 2003). To take the lower spectral resolution $(R=40000)$ into account, we rebined each spectrum with the bilinear approximation. Each speckle was sampled with a minimum of 9 pixels (greater than the Nyquist sampling) per resolution element. Then we obtained 9 spectra per $(\lambda / d)^{2}$. In Sect. 4.1, the AGPM coronagraph with a Lyot dot in the center gives the residual starlight by the $f_{\mathrm{R}}(\rho)$ function. In addition to the stellar shot noise, we take into account, the read-out noise (2e-/frame) (important due to a huge number of individual exposures $\left.>10^{5}-10^{6}\right)$, the dark noise $(0.001 \mathrm{e}-/ \mathrm{pix} / \mathrm{s})$, and finally the "flat" effect on each pixels ( $\pm 2 \%$ photometric deviation). This last effect is important as it corresponds to a different efficiency given by the IFU implementation on each spectrum. Contrary to Berton et al. (2006), we used the IFU implementation to provide radial velocity for the planet, not for the differential imaging technique. In these conditions, we keep spectral information of the planet. We note that the atmospheric scintillation is not taken into account in our simulation. Indeed, this effect in the differential mode (i.e. between each spectra) seems to be negligible in our application (Owner-Petersen 2006).

The spectrum is recorded in the 650 to $850 \mathrm{~nm}$ wavelength range with a spectroscopic resolution of 40000 (12000 pixels per spectrum). Assuming an Earth-like planet at $d_{p s}=$ $\sqrt{x x^{2}+y y^{2}}=1 \mathrm{AU}$, that corresponds to the radial velocity given by Eq. (10). To validate this method, we check the sensitivity in the 10 proposed stellar type and the two radial functions $f_{\mathrm{R}}(\rho) \approx 1300 / 3300$ given by the coronagraphic simulation (see Fig. 2).

In its present configuration, the ELT is a $42 \mathrm{~m}$ telescope with an overall efficiency of $15 \%$, all proposed stars are in the distance of $10 \mathrm{pc}$, and the Earth-like planet is $1 \mathrm{AU}$ of the parent star. We calculated the standard deviation of the multiple correlation (Eq. (12)) in a resolution element and on the full angular 


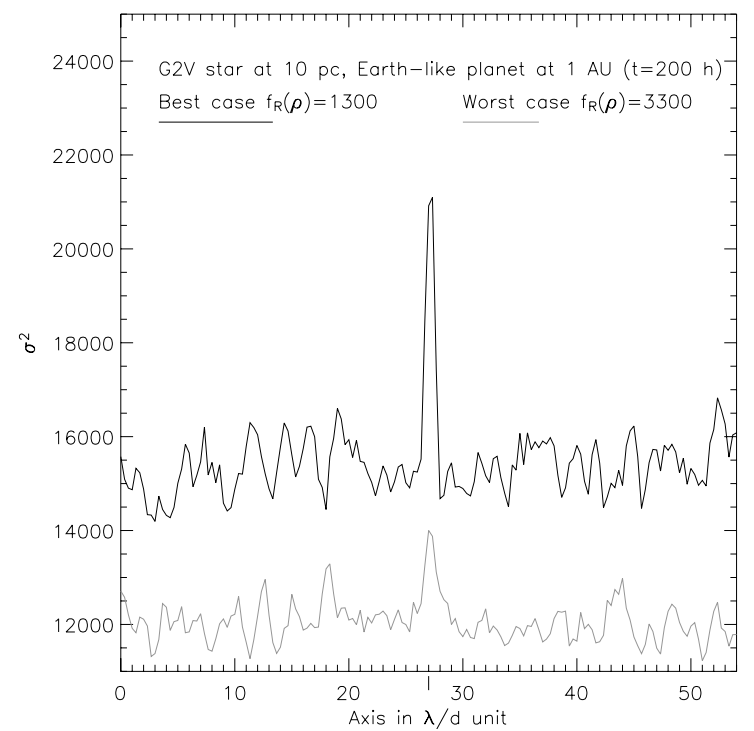

Fig. 6. Multiple correlations obtained on the G2V star at $10 \mathrm{pc}$. The number of spectra is 534, the sampling is 3 pixels per resel and an Earthlike planet is orbiting at $1 \mathrm{AU}$ (pixel number 81 with a telescope with a $42 \mathrm{~m}$ ). The total exposure time is $200 \mathrm{~h}$, and we correlate with all spectra. As we can see in Fig. 7, this example corresponds to worst planetary detection compared to all spectral types, except for the $M 5 \mathrm{~V}$ star. The exposure time is slightly longer than $150 \mathrm{~h}$ to have sufficient signal-to-noise ratio in the $f_{\mathrm{R}}(\rho)=3300$ case (grey line).

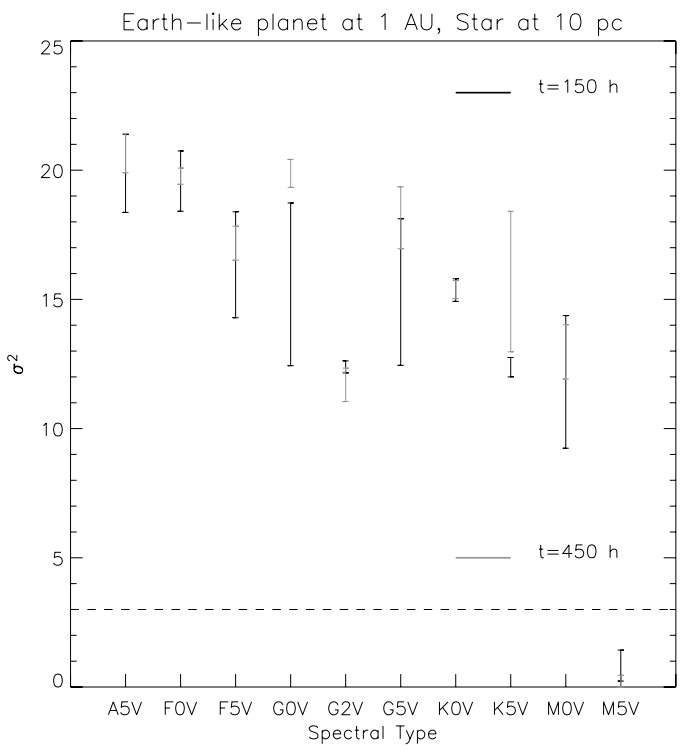

Fig. 7. Variance calculated for ten different stellar type stars. Each star is located at $10 \mathrm{pc}$ with the same Earth-like planet orbiting at $1 \mathrm{AU}$. Solid line, shows results for a total exposure time of 150 hours and grey line for $450 \mathrm{~h}$. The "error" bar present the signal to noise ratio for the two radial profiles $\left(f_{\mathrm{R}}(\rho)=1300-3300\right)$ obtained in the previous coronagraphic simulation. The correlation is obtained with all spectra. With a large spectrum correlation, we notice an improvement in the signal to noise ratio with a factor of about $N^{1 / 4}$, with $N$ the number of available spectra obtained by DRiVE.

separation with the $f_{\mathrm{R}}(\rho)$ function ( 0 to $0.267^{\prime \prime}$ or 534 pixels). Figures 5 and 6 show an example of multicorrelation where the correlation is done on the 534 spectra with $150 \mathrm{~h}$ of total exposure time.

After our first simulation obtained for an Earth-like planet at $1 \mathrm{AU}$ around nearby star at $10 \mathrm{pc}$ to validate the method, we

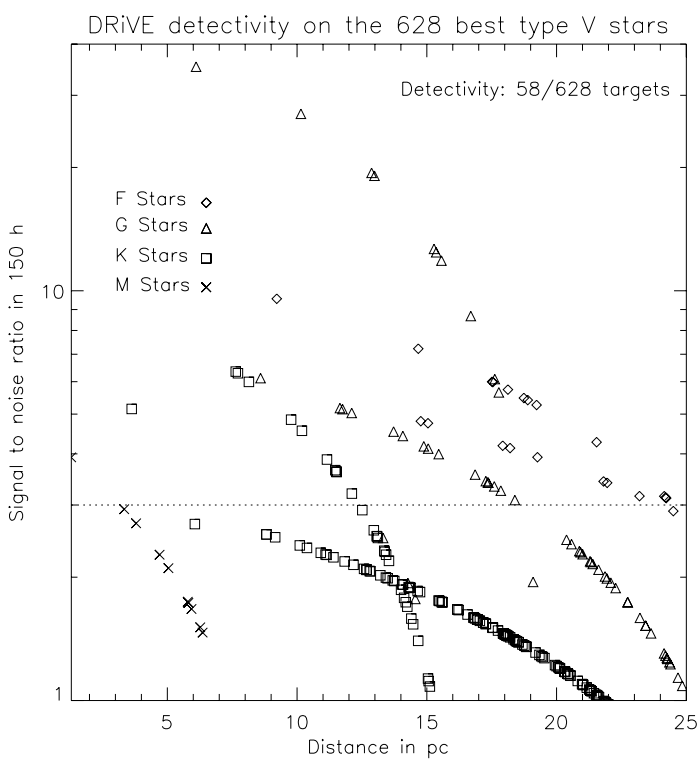

Fig. 8. Signal-to-noise ratio calculated for 628 stars provided by the TPF/DARWIN targets. Depending on the stellar characteristics, we place an Earth-like planet in the habitable zone (0.13 to $4.27 \mathrm{AU})$. The total exposure time was $150 \mathrm{~h}$ and the average spectral resolution was 45000 between 650 and $850 \mathrm{~nm}$. Some planets were not detected due to the insufficient spectral resolution (the Doppler is less than 4 pixels) not because of the flux limitation. Other parameters also appear, like the broadened spectral features that reduce the DRiVE detectivity for cold stars M0V and M5V.

scaned all possible planets in the habitable zone (4.27 AU for a $A 5 V$ star to 0.13 AU for a $M 5 V$ star, see Riaud et al. 2002) and between 1.3 to $25 \mathrm{pc}$. For a clean detectivity analysis, it was necessary to weight all results with the real distance of nearby stars. Then, we used the TPF/DARWIN star targets (628 stars, Wallner et al. 2006; Kaltenegger \& Fridlund 2006; Velusamy et al. 2006). Figure 8 shows the signal-to-noise ratio obtained with the same instrumental condition as explained above.

The spectral resolution needed for a planet Doppler-shift detection must be up to 40000 . If high resolution spectra like UVES $(R \approx 120000)$ can be used, the detectivity will be increased owing to the higher number of iron lines present in the spectra despite the proportional signal decrease in each spectral resolution element. For some stars like K5V or M0V spectral types, it is possible to detect the planet around them if we use spectral resolution higher than 50000 . Indeed, due to low-mass stars, the planet shift is less than 4 pixels on the spectra and not allowing proper detection. In these conditions, we can optimize the spectral resolution with the spectral type. This configuration $(R \approx 40000)$ seems to be well-suited for planetary detection around A to K stars, as shows. This is also less stringent concerning the number of CCD chips (see Sect. 5).

\section{Discussion}

The DRiVE implementation allows Earth-like planets to be detected with 150 to $450 \mathrm{~h}$ total exposure time with extreme adaptive optics and an optimized coronographic device. Previous studies by Cavarroc et al. (2006) show no detection with only $\mathrm{XAO}$ and a coronagraphic instrument due to the limitative effect of no-common path errors in the optical trains. The radial velocy improves the telluric planet detection with large ground-based telescopes and also removes the confusion with background objects. Now it is necessary to discuss the possible instrumental 
solution for DRiVE. An IFU implementation in the previous section is chosen for a good throughput and a possible large number of spectra. In the general case, the ELT instrumentation requires a completely new design due to the huge number of resolution elements. In our case, a $1^{\prime \prime} \times 1^{\prime \prime}$ covered by an IFU high resolution spectrograph could provide more than 880000 spectra on 647 $4096 \times 4096$ detectors. This type of instrumentation will be certainly realized for extragalactic reasearch like rotation curves for highly redshifted galaxies, full stellar population analysis, isolating HII regions, etc.

DRiVE will be able to use the existing high resolution spectrograph on the ELT, if we include an optimized coronagraphic mask for proper attenuation of the star. We also propose to use DRiVE for a powerful analysis of the host galaxy around Quasars and AGN. Concerning planet detection, it is possible to reduce the FOV of the IFU to a crown centered on the habitable zone of the star. For example, around a F5V star at $10 \mathrm{pc}$, the habitable zone could be studied with 5800 different spectra on the crown at $189 \pm 4$ mas of the star. This large number of spectra corresponds to four $4096 \times 4096$ detectors on the spectrograph. Generally speaking, our approach requires studing only a fine crown around the habitable zone distance; the width is provided by the stellar type of the target, and this zone is thinner for cooler stars ( $\mathrm{K}$ to $\mathrm{M}$ types). Finally, spectroscopic implementation could be highly simplified, if we know the planet position already detected by space-based instruments like TPF/DARWIN. We used only four different positions, one around the planet peak, and three symmetrical positions relative to the coronagraph to insure a proper speckle calibration issue. In this condition, less than 160 spectra can be simultaneously recorded on the $2048 \times 2048$ detector.

In addition, the DRiVE instrument will help to detect the oxygen $A$-band at $765 \mathrm{~nm}$ on exo-Earths. This band is about $10 \mathrm{~nm}$ wide, and its detection will be very difficult from the ground due to blending with the same band present in the Earth atmosphere. But this band has a fine structure with $\delta \lambda=0.1 \mathrm{~nm}$ wide features (Fournier et al. 2006). This fine structure allows the monitoring of the Doppler-shift $\Delta \lambda \approx 2 \times 30 \mathrm{~km} \mathrm{~s}^{-1} / c \times$ $765 \mathrm{~nm}=0.15 \mathrm{~nm}$ along the orbital motion of the Earth and the exoplanet. In our next paper we will present a complete simulation of the oxygen $A$-band detectability.

\section{Conclusions}

The DRiVE implementation allows Earth-like planet detection with an appropriate coronagraphic device mounted on the ELT. This detection in the visible wavelength is complementary to space-based detection like the TPF/DARWIN mission. Indeed, the knowledge of the planet position given by the space mission in the thermal infrared, for example, simplifies the spectrographic implementation on the ELT. Then, we reduce the observational field of view in 10 mas in radius centered on the planet position. In these conditions, the synergism appears with futur space-based instrumentations and the ELT to characterize Earth-like planets around nearby stars both in the thermal infrared and visible. For example, the DRiVE instrument will help for detecting of the oxygen on exo-Earths (not a sign of life if it is detected alone, but in conjunction with other spectral features like $\mathrm{O}_{3}, \mathrm{H}_{2} \mathrm{O}, \mathrm{CO}_{2}, \mathrm{CH}_{4}$, etc.). The full completeness in terms of wavelength coverage allows us to remove any false positive biosignature detections inevitable in the high contrast imaging under a low signal-to-noise ratio. It is essential to join forces with ground-based and space-based high contrast imaging instruments to search for life on the telluric exoplanet.

Acknowledgements. P.R. acknowledges the financial support of the University of Liège, the Centre Spatial de Liège, particularly Denis Vandormael and Florence Defraigne and the "Agence Nationale de la Recherche" in France. We want to thank O. Absil and J. Baudrand for their help on the manuscript. This work also received the support of PHASE, the high angular resolution partnership between ONERA, Observatoire de Paris, CNRS, and University Denis Diderot Paris 7. The authors are also grateful to the anonymous referee who helped improve the manuscript.

\section{References}

Arnold, L., Gillet, S., Lardière, O., Riaud, P., \& Schneider, J. 2002, A\&A, 392, 231

Bagnulo, S., Jehin, E., Ledoux, C., et al. 2003, Messenger, 114, 10

Berton, A., Gratton, R. G., Feldt, M., et al. 2006, PASP, 118, 1144

Brunetto, E. T., Dierickx, P., Gilmozzi, R., et al. 2004, in Emerging Optoelectronic Applications, ed. A. L. Ardeberg, \& T. Andersen, Proc. SPIE, 5382, 159

Bruston, P. 1992, Adv. Space Res., 12, 177

Cavarroc, C., Boccaletti, A., Baudoz, P., Fusco, T., \& Rouan, D. 2006, A\&A, 447, 397

Fournier, N., Stammes, P., de Graaf, M., et al. 2006, Atmos. Chem. Phys., 6, 163 Kaltenegger, L. \& Fridlund, M. 2006, in Direct Imaging of Exoplanets: Science \& Techniques, ed. C. Aime \& F. Vakili, IAU Coll., 200, 255

Le Louarn, M., Verinaud, C., Yaitskova, N., et al. 2004, in Advancements in Adaptive Optics, ed. D. Bonaccini Calia, B. L. Ellerbroek, \& R. Ragazzoni, Proc. SPIE, 5490, 649

Lovis, C., Pepe, F., Bouchy, F., et al. 2006, in Ground-based and Airborne Instrumentation for Astronomy, ed. I. S. McLean, \& M. Iye, Proc. SPIE, 6269, 62690

Lyot, B. 1939, MNRAS, 99, 538

Mawet, D., Riaud, P., Absil, O., \& Surdej, J. 2005, ApJ, 633, 1191

Owner-Petersen, M. 2006, in Advances in Adaptive Optics II, ed. B. L. Ellerbroek, \& D. Bonaccini Calia, Proc. SPIE, 6272, 62722

Pepe, F., Rupprecht, G., Avila, G., et al. 2003, in Instrument Design and Performance for Optical/Infrared Ground-based Telescopes, ed. M. Iye, \& A. F. M. Moorwood, Proc. SPIE, 4841, 1045

Prieto, E. \& Vivès, S. 2006, in Optomechanical Technologies for Astronomy, ed. Eli Atad-Ettedgui, J. Antebi, and Dietrich Lemke, Proc. SPIE, 6273, 627319 Riaud, P., Boccaletti, A., Rouan, D., Lemarquis, F., \& Labeyrie, A. 2001, PASP, 113,1145

Riaud, P., Boccaletti, A., Gillet, S., et al. 2002, A\&A, 396, 345

Velusamy, T., Marsh, K. A., \& Ware, B. 2006, in Direct Imaging of Exoplanets: Science \& Techniques, ed. C. Aime \& F. Vakili, IAU Coll., 200, 621

Vérinaud, C., Hubin, N., Kasper, M., et al. 2006, in Advances in Adaptive Optics II, ed. B. L. Ellerbroek, \& D. Bonaccini, Calia. Proc. SPIE, 6272, 627209

Wallner, O., Ergenzinger, K., Flatscher, R., \& Johann, U. 2006, in Advances in Stellar Interferometry, ed. J. D. Monnier, M. Schöller, W. C. Danchi, Proc. SPIE, 6268

Zamkotsian, F., Waldis, S., Noell, W., et al. 2006, in Optomechanical Technologies for Astronomy, ed. Eli Atad-Ettedgui, J. Antebi, \& D. Lemke, Proc. SPIE, 6273, 627319 